\title{
论 文庆祝朱道本院士80华诞专刊
}

\section{二维石墨炔/金属氢氧化物异质结用于高效析氧 反应}

高雅奇 ${ }^{1}$, 薛玉瑞 ${ }^{*}$, 李玉良 ${ }^{1,2 *}$

1. 山东大学物质创制与能量转化科学研究中心, 化学与化工学院, 济南 250100

2. 中国科学院化学研究所, 北京 100190

*通讯作者, E-mail: xueyurui@iccas.ac.cn; ylli@iccas.ac.cn

收稿日期: 2021-08-31; 接受日期: 2021-10-09; 网络版发表日期: 2021-10-28

国家重点研发计划(编号: 2018YFA0703501)、国家自然科学基金(编号: 21790050, 21790051)、山东省自然科学基金重大基础研究项目(编号: ZR2020ZD38)和山东省泰山学者工程青年专家计划(编号: tsqn201909050)资助项目

摘要析氧反应(OER)是能量转换过程中重要的半反应, 开发高效、稳定、低成本的析氧反应催化剂具有重要 的意义. 本文报道了一种简单的原位可控生长的方法, 在铜双金属氢氧化物纳米片表面原位生长石墨炔薄膜, 形 成了新型石墨炔/金属氢氧化物异质结界面结构, 并通过改变化学组成的方法, 实现对其电催化OER活性和稳定 性的有效提升. 研究结果显示 $\mathrm{Ni}_{0.74} \mathrm{Cu}_{0.26} \mathrm{LDH} @ \mathrm{GDY} / \mathrm{NF}$ 具有最佳 $\mathrm{OER}$ 催化性能. 电流密度为 $10 \mathrm{~mA} \mathrm{~cm}^{-2}$ 时的过 电位仅为 $292 \mathrm{mV}$, 且具有优异的长期稳定性, 比如在 6000 圈连续的循环测试后 OER活性几乎无衰减. 实验结果表 明, 石墨炔的引入可以有效增加活性表面积和活性位点, 促进电荷转移提高导电性, 且能够高效保护催化剂不被 腐蚀, 从而提高整体催化性能.

关键词石墨炔, 异质结催化剂, 双金属氢氧化物, 析氧反应

\section{1 引言}

析氧反应 $(\mathrm{OER})$ 是许多新能源转换技术(比如, 电 解水、 $\mathrm{CO}_{2}$ 还原、金属-空气电池等) 重要的半反 应 $^{[1 \sim 10]}$. 然而, 缓慢的反应动力学限制了 OER催化性能 的提升 ${ }^{[2,3,11 ~ 13]}$. 目前报道的具有优异OER催化性能的 催化剂主要为贵金属基材料 $\left(\mathrm{RuO}_{2} 、 \mathrm{IrO}_{2}\right.$ 等 $)$, 但是贵 金属自身的储量低、稳定性差等因素严重限制了贵金 属基OER催化剂的实际应用 ${ }^{[13]}$. 因此，设计与合成具 有高反应活性的OER催化剂变得尤为重要，成为提升 能源转换器件效率的关键. 过渡金属元素因其物产丰
富，且拥有多变价活性中心，受到了研究者的广泛关 注. 经过科学者们的不解努力, 诸多过渡金属基氧化 物、磷化物、硫化物、硒化物、羟基氧化物等OER电 催化剂已被报道. 水滑石 (LDH) 是一种层状双金属氢 氧化物，在催化领域具有巨大的应用潜力 ${ }^{[14 \sim 20]}$. 总结 众多的水滑石电催化剂, 我们不难发现, 反应活性 低、稳定性差成为制约其发展的重要瓶颈.

石墨炔(graphdiyne, GDY, 由李玉良院士第一次汉 语命名为石墨炔)是一种由 $\mathrm{sp}$ 和 $\mathrm{sp}^{2}$ 杂化碳构成的新型 二维平面碳材料, 具有诸多独特的结构性质, 比如丰富 的碳化学键、天然的孔洞结构、高导电性和优异的化

引用格式: Gao Y, Xue Y, Li Y. Two-dimensional graphdiyne/metal hydroxide heterojunction for high-efficiency oxygen evolution reaction. Sci Sin Chim, 2022, 52: 321-329, doi: 10.1360/SSC-2021-0203 
学稳定性 ${ }^{[21 ~ 23]}$. 最重要的是, 其表面电荷不均匀分布 可引起高本征活性，同时它是唯一一种可以在低温常 压条件下可控合成的碳材料，改变了传统碳材料单一 的杂化方式和高温高压等的制备方法，在生长、组装 和性能调控等方面表现出巨大优势和先进性，可衍生 出具有明确化学结构和电子结构的新材料, 引发材料 研究的新现象和新知识, 为科学创新提供了无限空 间 ${ }^{[24-29]}$. 自2010年由中国科学家李玉良院士课题组首 次成功合成以来, 石墨炔在催化、能源、光学、电学 等领域展现了巨大的应用潜力，在提升催化剂活性和 稳定性等方面具有突出优势 ${ }^{[30 \sim 47]}$.

在本文中，我们通过简单的原位生长的方法成功 制备了石墨炔/铜双氢氧化物异质结催化剂, 并将其成 功应用于电催化OER反应中. 研究结果显示, 在碱性环 境中, 该催化剂具有优异的析氧催化活性. 通过可控调 节催化剂中 $\mathrm{Ni} / \mathrm{Cu}$ 比例, 可以有效调控催化剂的形貌、 电子结构和催化性能. 电化学测试结果显示, 当 $n_{\mathrm{Ni}}: n_{\mathrm{Cu}}=0.74: 0.26$ 时, 该催化剂 $\left(\mathrm{Ni}_{0.74} \mathrm{Cu}_{0.26} \mathrm{LDH} @ \mathrm{GDY} /\right.$ $\mathrm{NF}$ )具有最佳的OER催化活性和催化稳定性.

\section{2 实验部分}

\section{1 试剂与仪器}

硫酸镍六水合物、尿素、氢氧化钾、盐酸和无水 乙醇购自国药集团试剂有限公司; 三水硝酸铜购自 $3 \mathrm{~A}$ 试剂有限公司; 氟化铵购自麦克林试剂有限公司. 所有 使用的试剂均为分析纯. 超纯水采用Millipore 超纯水 系统制备.

\section{2 催化剂的制备及其表征}

将清洗干净的 $2 \mathrm{~cm} \times 2 \mathrm{~cm}$ 泡沫镍置于含有 $0.25 \mathrm{mmol} \mathrm{Cu}\left(\mathrm{NO}_{3}\right)_{2} \cdot 3 \mathrm{H}_{2} \mathrm{O}, 2.25 \mathrm{mmol} \mathrm{Ni}\left(\mathrm{SO}_{4}\right)_{2} \cdot 6 \mathrm{H}_{2} \mathrm{O}$, $3 \mathrm{mmol}$ 尿素和7.5 mmol $\mathrm{NH}_{4} \mathrm{~F}$ 的 $30 \mathrm{~mL}$ 超纯水溶液中, 于 $120^{\circ} \mathrm{C}$ 烘箱里水热反应 12 小时. 反应结束后, 将所得 样品依次用去离子水和无水乙醇冲洗，最后在 $60^{\circ} \mathrm{C}$ 的 真空干燥箱中干燥 12 小时, 得到 $\mathrm{Ni}_{0.9} \mathrm{Cu}_{0.1} \mathrm{LDH} / \mathrm{NF}$. 通 过改变 $\mathrm{Cu}\left(\mathrm{NO}_{3}\right)_{2} \cdot 3 \mathrm{H}_{2} \mathrm{O}$ 与 $\mathrm{Ni}\left(\mathrm{SO}_{4}\right)_{2} \cdot 6 \mathrm{H}_{2} \mathrm{O}$ 的摩尔比来制 备不同比例的 $\mathrm{Ni}_{x} \mathrm{Cu}_{1-x} \mathrm{LDH} / \mathrm{NF}(0 \leq x \leq 1)$.

将新制的 $\mathrm{Ni}_{0.9} \mathrm{Cu}_{0.1} \mathrm{LDH} / \mathrm{NF}$ 样品放入 $30 \mathrm{~mL}$ 溶有六乙 炔基苯(HEB)的吡啶溶液中, 然后将溶液转移到 $50 \mathrm{~mL}$ 聚四氟乙烯内祄的不锈钢高压反应釜中, 在 $110^{\circ} \mathrm{C}$ 下反
应 $10 \mathrm{~h}$. 反应后, 依次用热的二甲基甲酰胺和丙酮洗涤 三次, 然后在 $60^{\circ} \mathrm{C}$ 的真空干燥箱中干燥, 即可得到 $\mathrm{Ni}_{0.9^{-}}$ $\mathrm{Cu}_{0.1} \mathrm{LDH} @ \mathrm{GDY} / \mathrm{NF}$ 及系列浓度比例 $\mathrm{Ni}_{x} \mathrm{Cu}_{1-x} \mathrm{LDH} @$ $\operatorname{GDY} / \mathrm{NF}(0 \leq x \leq 1)$ 样品.

将制备的电极材料用冷场发射扫描电镜( S- 4800 , FESEM, 日本日立公司, 加速电压 $3 \mathrm{kV}$ )观测其形貌结 构, 能谱仪(EDX)进行元素组成及分布测试(测试电压 $5 \mathrm{kV})$. 采用X射线衍射仪 (SmartLab, $9 \mathrm{~kW}, \mathrm{XRD}$, 日本 理学公司)进行电极样品的晶型结构的测试. 用低倍冷 场发射透射电子显微镜(JEM-1011，TEM，工作电压 $100 \mathrm{kV})$ 和高分辨透射电子显微镜 $(\mathrm{JEM}-2100 \mathrm{~F}$, HRTEM, 工作电压 $200 \mathrm{kV}$ )来表征样品形态的详细信 息. 用X射线光电子能谱仪 (XPS, Thermo Fisher ESCA$\mathrm{LAB} \mathrm{XI}+$ )测定催化剂的表面离子价态 $\left(\mathrm{Al}, K_{\alpha}\right)$. 用拉曼 光谱仪(Renishaw-2000, 激发光源波长为473 nm) 测得 样品的拉曼光谱.

\section{3 电化学测试}

采用标准的三电极体系, 在 $\mathrm{CHI} 660 \mathrm{E}$ (上海, 辰华) 电化学工作站上进行相关催化剂催化性能测试. 新鲜 制备的催化剂作为工作电极, 碳棒作为对电极, 饱和 甘录电极(SCE)作为参比电极, 氧气饱和的 $1.0 \mathrm{~mol} \mathrm{~L}^{-1}$ $\mathrm{KOH}$ 作为电解液. 本文中, 所有未注明的电极电势均 根据公式 $\left(E_{(\mathrm{RHE})}=E_{(\mathrm{SCE})}+E_{(\mathrm{SCE})}^{0}+0.05916 \times \mathrm{pH}\right)$ 转换为标 准氢电极电势. 在析氧反应中, 线性扫描伏安法(LSV) 的扫描窗口是 $0 \sim 1 \mathrm{~V}\left(v s\right.$. SCE), 扫描速度是 $2 \mathrm{mV} \mathrm{s}^{-1}$. 循环伏安 $(C V)$ 测试时, 扫描速度为 $100 \mathrm{mV} \mathrm{s}^{-1}$. 电化学 阻抗谱(EIS)测试的频率区间设置为 $100 \mathrm{kHz} 0.01 \mathrm{~Hz}$. 双电层电容 $\left(C_{\mathrm{d} 1}\right)$ 测试采用 $\mathrm{CV}$ 法在非法拉第电流区间 测试, 工作电压区间为 $0 \sim 1 \mathrm{~V}$.

\section{3 结果与讨论}

\section{1 催化剂的制备及其表征}

催化剂的制备采用两步合成策略: 首先, 通过水 热法在泡沫镍基底上生长镍铜基双氢氧化物结构 $\left(\mathrm{Ni}_{x} \mathrm{Cu}_{1-x} \mathrm{LDH} / \mathrm{NF}, 0 \leq x \leq 1\right)$, 随后，以实现六乙炔基苯 (HEB)为前体分子在镍铜基双氢氧化物表面通过Glaser-Hay偶联反应原位温和可控生长一层石墨炔薄膜, 形成石墨炔包覆的异质结催化剂 $\left(\mathrm{Ni}_{x} \mathrm{Cu}_{1-x} \mathrm{LDH} @\right.$ GDY/NF, $0 \leq x \leq 1)$. 
我们首先利用扫描电子显微镜(SEM)、透射电子 显微镜(TEM)以及高分辨透射电镜(HRTEM)分别对所 制备的样品的形貌进行了详细表征. 如图1a所示, 泡沫 镍经预处理后表面光滑，无颗粒物存在，纯相单金属 $\mathrm{NiLDH}$ (图1b)为纳米片组装成的微米球结构. 在引入 少量 $\mathrm{Cu}$ 元素的情况下 $(1-x=0.1 \sim 0.4$, 图 $1 \mathrm{c} \sim \mathrm{g})$, 所得催化 剂基本保持微米球形貌结构, 该微米球大小发生改变, 以及组装成微米球的纳米片的形貌均随着 $\mathrm{Cu}$ 加入量 的改变而发生改变. 当进一步增加 $\mathrm{Cu}$ 元素含量时(1$x=0.5 \sim 1.0$, 图 $1 \mathrm{~h} \sim 1)$, 所得催化剂的形状变的不规则, 甚 至呈团聚状.

图2为包覆石墨炔后的催化剂的扫描电镜图片. 如 图所示, 相比于未包覆石墨炔样品的光滑的表面(图1), $\mathrm{NiLDH} @$ GDY/NF、 $\mathrm{Ni}_{0.74} \mathrm{Cu}_{0.26} \mathrm{LDH} @$ GDY/NF、 CuLDH@GDY/NF表面均呈现出明显的褶皱，表面变 得更加粗粘, 表明石墨炔在其表面成功生长. 图 $2 \mathrm{~d} \sim \mathrm{f}$
显示, 相比于纯的NiLDH@GDY/NF和CuLDH@GDY/ NF样品, $\mathrm{Ni}_{0.74} \mathrm{Cu}_{0.26} \mathrm{LDH} @ \mathrm{GDY} / \mathrm{NF}$ 球状结构具有更加 细致精巧的二级结构, 在微米球表面遍布众多由纳米 薄片组装而成的“脊”. 与 $\mathrm{Ni}_{0.74} \mathrm{Cu}_{0.26} \mathrm{LDH} / \mathrm{NF}$ 相比, $\mathrm{Ni}_{0.74} \mathrm{Cu}_{0.26} \mathrm{LDH} @ \mathrm{GDY} / \mathrm{NF}$ 保持了原始的微米球形貌, 表明在石墨炔原位包覆过程中并未对金属氢氧化物的 形貌产生破坏, 也表明该催化剂结构的高稳定性. 高倍 扫描电子显微镜 $(S E M)$ 照片显示, 纳米片表面变得更 加粗粘，表明了石墨炔的存在，同时也意味着石墨炔 的引入能够增加其比表面积. 透射电子显微镜(图3a, b)表明催化剂微米球结构是由纳米薄片有序组装形 成. 图 $3 \mathrm{c}, \mathrm{d}$ 为 $\mathrm{Ni}_{0.74} \mathrm{Cu}_{0.26} \mathrm{LDH} @ \mathrm{GDY} / \mathrm{NF}$ 纳米片的高分 辨透射电镜图, 同时观察到了金属氢氧化物与石墨炔, 包覆的石墨炔厚度为 $6.83 \mathrm{~nm}$ ，也进一步表明石墨炔基 异质结构的成功构筑.

随后我们利用 $X$ 射线衍射 $(X R D)$ 、拉曼和 $X$ 射线
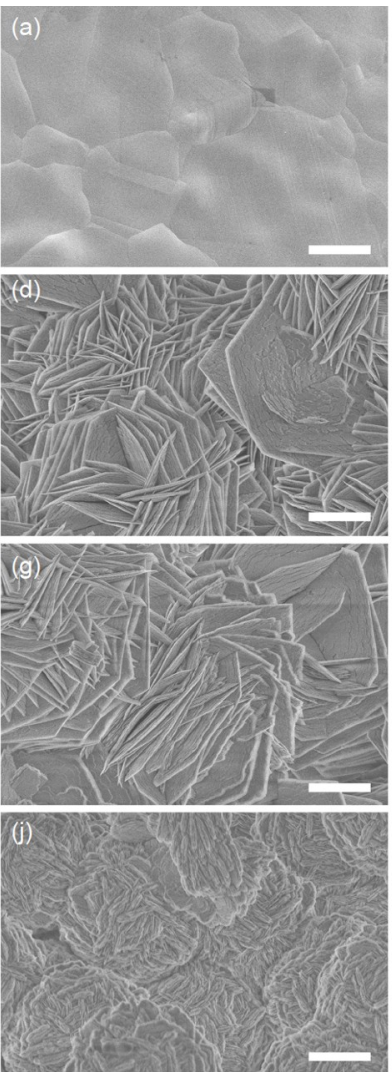
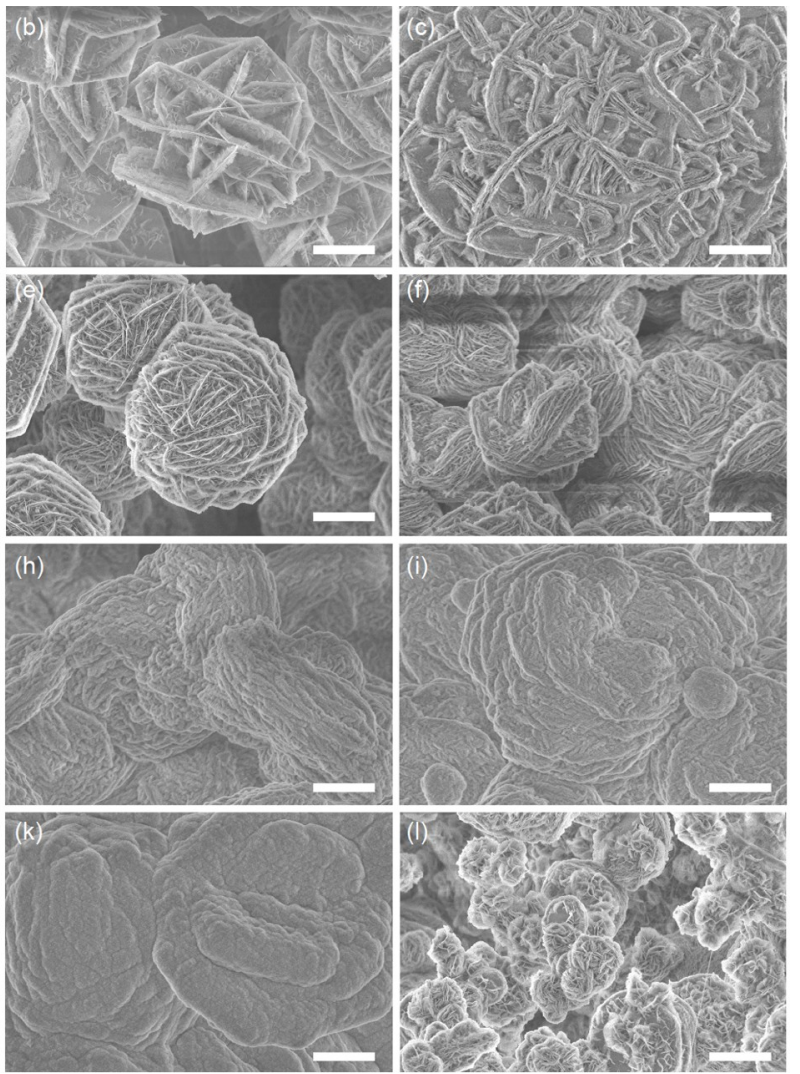

图 1 (a) 泡沫镍; (b) NiLDH/NF; (c) $\mathrm{Ni}_{0.9} \mathrm{Cu}_{0.1} \mathrm{LDH} / \mathrm{NF}$; (d) $\mathrm{Ni}_{0.8} \mathrm{Cu}_{0.2} \mathrm{LDH} / \mathrm{NF}$; (e) $\mathrm{Ni}_{0.74} \mathrm{Cu}_{0.26} \mathrm{LDH} / \mathrm{NF}$; (f) $\mathrm{Ni}{ }_{0.7} \mathrm{Cu}{ }_{0.3} \mathrm{LDH} / \mathrm{NF}$; (g) $\mathrm{Ni}_{0.6} \mathrm{Cu}_{0.4} \mathrm{LDH} / \mathrm{NF}$; (h) $\mathrm{Ni}_{0.5} \mathrm{Cu}_{0.5} \mathrm{LDH} / \mathrm{NF}$; (i) $\mathrm{Ni}_{0.4} \mathrm{Cu}_{0.6} \mathrm{LDH} / \mathrm{NF}$; (j) $\mathrm{Ni}_{0.3} \mathrm{Cu}_{0.7} \mathrm{LDH} / \mathrm{NF}$; (k) $\mathrm{Ni}_{0.1} \mathrm{Cu}{ }_{0.9} \mathrm{LDH} / \mathrm{NF}$; (l) $\mathrm{CuLDH} / \mathrm{NF}$ 的 SEM图. 标尺: $3 \mu \mathrm{m}$

Figure 1 SEM images of (a) Ni foam; (b) NiLDH/NF; (c) $\mathrm{Ni}_{0.9} \mathrm{Cu}_{0.1} \mathrm{LDH} / \mathrm{NF}$; (d) $\mathrm{Ni}_{0.8} \mathrm{Cu}_{0.2} \mathrm{LDH} / \mathrm{NF}$; (e) $\mathrm{Ni}_{0.74} \mathrm{Cu}_{0.26} \mathrm{LDH} / \mathrm{NF}$; (f) Ni ${ }_{0.7} \mathrm{Cu} 0.3 \mathrm{LDH} / \mathrm{NF}$; (g) $\mathrm{Ni}_{0.6} \mathrm{Cu}_{0.4} \mathrm{LDH} / \mathrm{NF}$; (h) $\mathrm{Ni}_{0.5} \mathrm{Cu}_{0.5} \mathrm{LDH} / \mathrm{NF}$; (i) $\mathrm{Ni}_{0.4} \mathrm{Cu}_{0.6} \mathrm{LDH} / \mathrm{NF}$; (j) $\mathrm{Ni}_{0.3} \mathrm{Cu}_{0.7} \mathrm{LDH} / \mathrm{NF}$; (k) $\mathrm{Ni}_{0.1} \mathrm{Cu}_{0.9} \mathrm{LDH} / \mathrm{NF}$; (l) CuLDH/NF. Scale bars: $3 \mu \mathrm{m}$. 

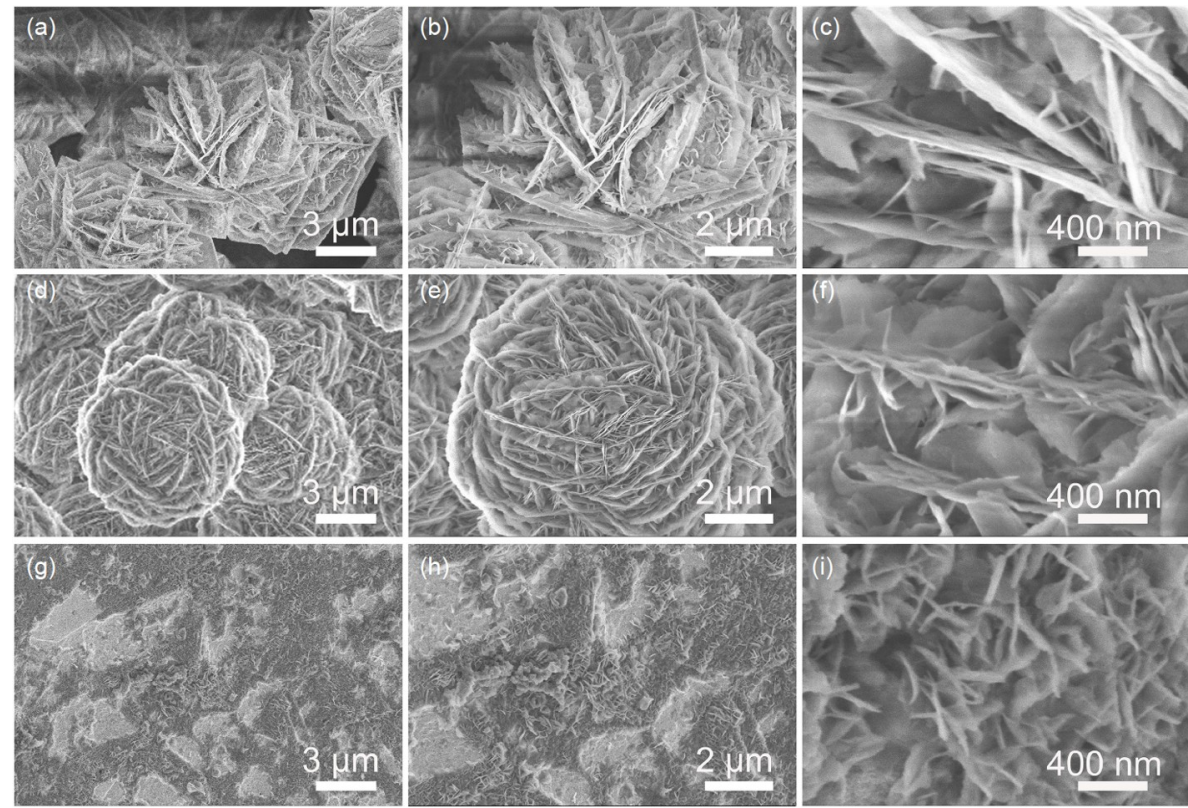

图 $2(\mathrm{a} \sim \mathrm{c}) \mathrm{NiLDH} @ \mathrm{GDY} / \mathrm{NF} 、(\mathrm{~d} \sim \mathrm{f}) \mathrm{Ni}_{074} \mathrm{Cu}_{026} \mathrm{LDH} @ G D Y / N F$ 和( $\left.\mathrm{g} \sim \mathrm{i}\right) \mathrm{CuLDH} @$ GDY/NF的SEM图

Figure 2 SEM images of (a-c) NiLDH@GDY/NF, (d-f) Ni $\mathrm{i}_{0.74} \mathrm{Cu}_{0.26} \mathrm{LDH} @ G D Y / N F,(\mathrm{~g}-\mathrm{i}) \mathrm{CuLDH} @ G D Y / N F$.

光电子能谱(XPS)测试分别对样品的结构组成进行了 测试分析. XRD测试结果(补充图1)显示, $\mathrm{Ni}_{0.74} \mathrm{Cu}_{0.26}$ LDH@GDY/NF具有良好的结晶性. 拉曼光谱测试结 果显示(图4a)，纯石墨炔样品在1371.5、1594.9、 1917.0、2181.8 $\mathrm{cm}^{-1}$ 处存在四个峰，其中 1371.5 和 $1594.9 \mathrm{~cm}^{-1}$ 处的两个峰分别对应 $\mathrm{D}$ 带和 $\mathrm{G}$ 带, 另外两个 峰则对应于石墨炔中三键的特征振动峰. 在 $\mathrm{Ni}_{0.74} \mathrm{Cu}_{0.26}$ LDH@GDY/NF的拉曼光谱中，1387.6、1601.2、
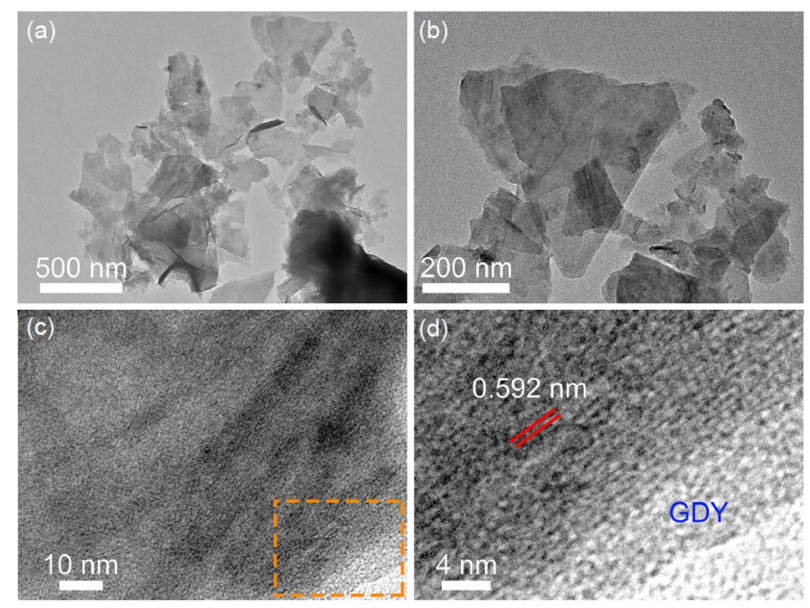

图 $3 \mathrm{Ni}_{0.74} \mathrm{Cu}_{0.26} \mathrm{LDH} @ \operatorname{GDY} / \mathrm{NF}$ 的(a, b) TEM和(c, d) HRTEM图 (网络版彩图)

Figure 3 (a, b) TEM and (c, d) HRTEM images of $\mathrm{Ni}_{0.74} \mathrm{Cu}_{0.26}$ LDH@GDY/NF (color online).
1946.3、2119.2 $\mathrm{cm}^{-1}$ 处的四个峰证明了石墨炔在 $\mathrm{Ni}_{0.74^{-}}$ $\mathrm{Cu}_{0.26} \mathrm{LDH} / \mathrm{NF}$ 表面的成功生长包覆. 如图 $4 \mathrm{~b}$ 所示, XPS 全谱分析结果显示 $\mathrm{Ni}_{0.74} \mathrm{Cu}_{0.26} \mathrm{LDH} @ G \mathrm{GDY} / \mathrm{NF}$ 中包含 C、O、Ni和Cu四种元素. Ni 2p XPS谱(图4c)显示, $\mathrm{Ni}_{0.74} \mathrm{Cu}_{0.26} \mathrm{LDH} @$ GDY/NF在 855.98、857.39、 873.64、862.01、862.01和 $880.01 \mathrm{eV}$ 处存在六个峰, 分 别归属于 $\mathrm{Ni} 2 \mathrm{p}_{2 / 3}$ 和 $\mathrm{Ni} 2 \mathrm{p}_{1 / 2}$ 及其相应的卫星峰. 精细分 峰拟合结果证明了 $\mathrm{Ni}_{0.74} \mathrm{Cu}_{0.26} \mathrm{LDH} @ \mathrm{GDY} / \mathrm{NF}$ 的金属 镍为 $\mathrm{Ni}^{2+}$ 和 $\mathrm{Ni}^{3+}$ 两种价态. 在 $\mathrm{Ni}_{0.74} \mathrm{Cu}_{0.26} \mathrm{LDH} @$ GDY/NF 的 $\mathrm{Cu} 2 \mathrm{p}$ 的放大谱(图4d)中共存在 8 个峰, 其中结合能 在 932.93 和 $952.98 \mathrm{eV}$ 处的特征峰对应于 $\mathrm{Cu} 2 \mathrm{p}_{3 / 2}$ 和 $\mathrm{Cu} 2 \mathrm{p}_{1 / 2}$, 揭示了 $\mathrm{Cu}^{+}$的存在; 结合能在 935.17 和 $954.92 \mathrm{eV}$ 处的特征峰对应于 $\mathrm{Cu} 2 \mathrm{p}_{3 / 2}$ 和 $\mathrm{Cu} 2 \mathrm{p}_{1 / 2}$, 揭示 了 $\mathrm{Cu}^{2+}$ 的存在. $\mathrm{Cu} 2 \mathrm{p}$ 和Ni $2 \mathrm{p}$ 的XPS结果都显示, 在 $\mathrm{Ni}_{0.74} \mathrm{Cu}_{0.26} \mathrm{LDH} @ G D Y / \mathrm{NF}$ 催化剂中的金属都具有混 合价态，该混合价态存在将有益于生成新的活性位点， 同时改善催化剂的电荷转移行为, 提升其催化性能. 此 外, 与纯 $\mathrm{Ni}_{0.74} \mathrm{Cu}_{0.26} \mathrm{LDH} / \mathrm{NF}$ 相比, $\mathrm{Ni}_{0.74} \mathrm{Cu}_{0.26}$ LDH@GDY/NF催化剂的 $\mathrm{Ni} 2 \mathrm{p}$ 和 Cu 2p结合能发生移 动，这表明石墨炔和金属氢氧化物之间存在明显的电 荷转移 ${ }^{[33]} . \mathrm{Ni}_{0.74} \mathrm{Cu}_{0.26} \mathrm{LDH} @$ GDY/NF的XPS C 1s 光谱 (补充图3)在284.53 eV ( $\left.\mathrm{sp}^{2}-\mathrm{C}\right) 、 285.1 \mathrm{eV} \quad(\mathrm{sp}-\mathrm{C}) 、$ $286.3 \mathrm{eV}(\mathrm{C}-\mathrm{O}) 、 288.24 \mathrm{eV}(\mathrm{C}=\mathrm{O})$ 处存在四个峰, 且 

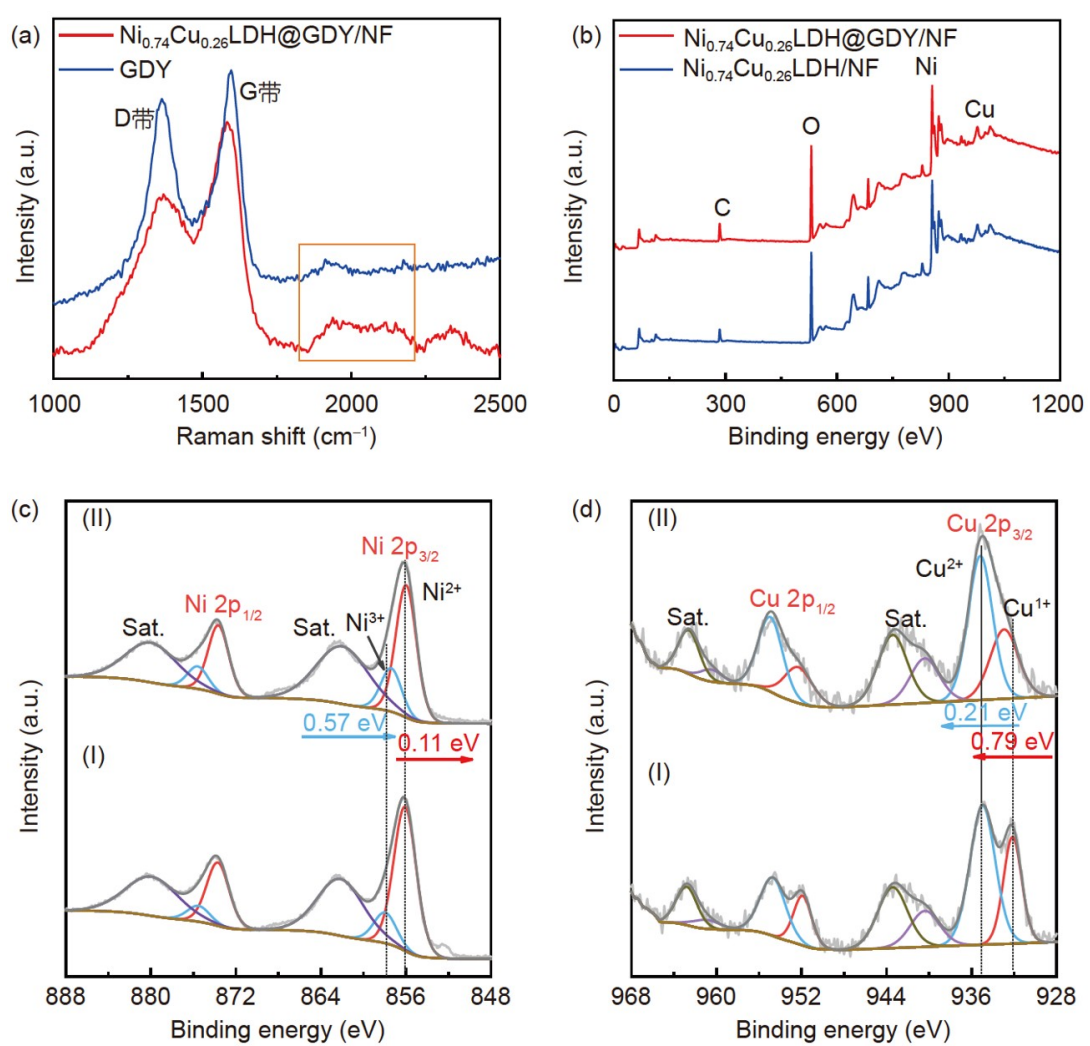

图 4 (a) $\mathrm{Ni}_{0.74} \mathrm{Cu}_{0.26} \mathrm{LDH} @ G D Y / N F$ 和GDY的拉曼光谱; $\mathrm{Ni}_{0.74} \mathrm{Cu}_{0.26} \mathrm{LDH} / \mathrm{NF}$ 和 $\mathrm{Ni}_{0.74} \mathrm{Cu}_{0.26} \mathrm{LDH} @ G D Y / \mathrm{NF}$ 样品的 (b) XPS全谱, (c) $\mathrm{Ni} 2$ p谱和 (d) $\mathrm{Cu} 2 p$ 谱: (I) $\mathrm{Ni}_{0.74} \mathrm{Cu}_{0.26} \mathrm{LDH} / \mathrm{NF}$ 和(II) $\mathrm{Ni}_{0.74} \mathrm{Cu}_{0.26} \mathrm{LDH} @$ GDY/NF (网络版彩图)

Figure 4 (a) Raman spectra of $\mathrm{Ni}_{0.74} \mathrm{Cu}_{0.26} \mathrm{LDH} @$ GDY/NF and GDY. (b) XPS survey, (c) $\mathrm{Ni} 2 \mathrm{p}$, and (d) $\mathrm{Cu} 2 \mathrm{p}$ XPS spectra of (I) $\mathrm{Ni}_{0.74} \mathrm{Cu}_{0.26} \mathrm{LDH} /$ $\mathrm{NF}$ and (II) $\mathrm{Ni}_{0.74} \mathrm{Cu}_{0.26} \mathrm{LDH} @ \mathrm{GDY} / \mathrm{NF}$ (color online).

在 $293.1 \mathrm{eV}$ 处新出现一个 $\pi-\pi *$ 跃迁峰，这也表明石墨 炔和 LDH之间存在显著的电荷转移. 这些研究结果显 示, 石墨炔的引入将会显著改善催化剂的电荷转移行 为, 增强导电性, 且生成新的活性位点, 进而显著提高 催化剂的催化活性.

\section{2 催化剂的电化学性质表征}

该研究工作中, 我们采用标准的三电极体系对催 化剂的催化性能进行评价. 新制备的样品作为工作电 极, 碳棒作为对电极, 饱和甘录电极作为参比电极. 氧 气充分饱和的 $1.0 \mathrm{~mol} \mathrm{~L}^{-1} \mathrm{KOH}$ 溶液作为电解液.

如图5a所示, $\mathrm{Ni}_{0.74} \mathrm{Cu}_{0.26} \mathrm{LDH} @ \mathrm{GDY} / \mathrm{NF}$ 具有最优 异的析氧活性, 在 $10 \mathrm{~mA} \mathrm{~cm}$ 电流密度下, 过电位仅 为 $292 \mathrm{mV}$; 显著优于 $\mathrm{NiLDH} @$ GDY/NF $(414 \mathrm{mV})$ 、 CuLDH@GDY/NF (367 mV)、 $\mathrm{Ni}_{0.74} \mathrm{Cu}_{0.26} \mathrm{LDH} / \mathrm{NF}$ (356 mV)及其他 $\mathrm{Cu} / \mathrm{Ni}$ 比例等对照样品(补充表1). 电 化学测试结果显示, 通过调节 $\mathrm{Cu} / \mathrm{Ni}$ 比例可以有效调控
催化剂的OER活性. Tafel数值大小可揭示反应动力学 快慢及反应机制等关键性电催化参数. 图 $5 \mathrm{~b}$ 表明，与 $\mathrm{NiLDH} @$ GDY/NF $\left(115 \mathrm{mV} \mathrm{dec}{ }^{-1}\right) 、 \mathrm{Ni}_{0.74} \mathrm{Cu}_{0.26} \mathrm{LDH} /$ $\mathrm{NF}\left(147 \mathrm{mV} \mathrm{dec}{ }^{-1}\right.$ )相比, $\mathrm{Ni}_{0.74} \mathrm{Cu}_{0.26} \mathrm{LDH} @$ GDY/NF具 有更小的Tafel斜率 $\left(103 \mathrm{mV} \mathrm{dec}{ }^{-1}\right.$ ), 说明 $\mathrm{Ni}_{0.74} \mathrm{Cu}_{0.26}$ $\mathrm{LDH} @ \mathrm{GDY} / \mathrm{NF}$ 具有更快的反应动力学. 在碱性电解 质中, OER一般包括四个步骤: (I) $\mathrm{M}+\mathrm{OH}^{-} \rightarrow$ $\mathrm{M}-\mathrm{OH}_{\mathrm{ads}}{ }^{*}+\mathrm{e}^{-}$; (II) $\mathrm{M}-\mathrm{OH}_{\mathrm{ads}}{ }^{*} \rightarrow \mathrm{M}-\mathrm{OH}$ ads ; (III) $\mathrm{M}-\mathrm{OH}_{\text {ads }}+\mathrm{OH}^{-} \rightarrow \mathrm{M}-\mathrm{O}_{\text {ads }}+\mathrm{H}_{2} \mathrm{O}+\mathrm{e}^{-}$; (IV) $\mathrm{M}-\mathrm{O}_{\text {ads }} \rightarrow$ $\mathrm{M}+1 / 2 \mathrm{O}_{2} \cdot \mathrm{Ni}_{0.74} \mathrm{Cu}_{0.26} \mathrm{LDH} @$ GDY/NF的Tafel斜率在 60 120 mV dec ${ }^{-1}$ 之间, 这表明该电极上的决速步骤与 步骤III和步骤IV都有关. 此外, 与纯的金属氢氧化物相 比, 石墨炔的包覆能够显著提高OER活性, 也进一步证 明石墨炔基异质结构的形成带来了更多数量的活性位 点, 进而提高其催化活性[48].

电化学阻抗谱(EIS)测试被用来分析催化剂的电 子和电荷转移行为. 在电化学阻抗谱(图 $5 \mathrm{c}$ )中, 半圆的 

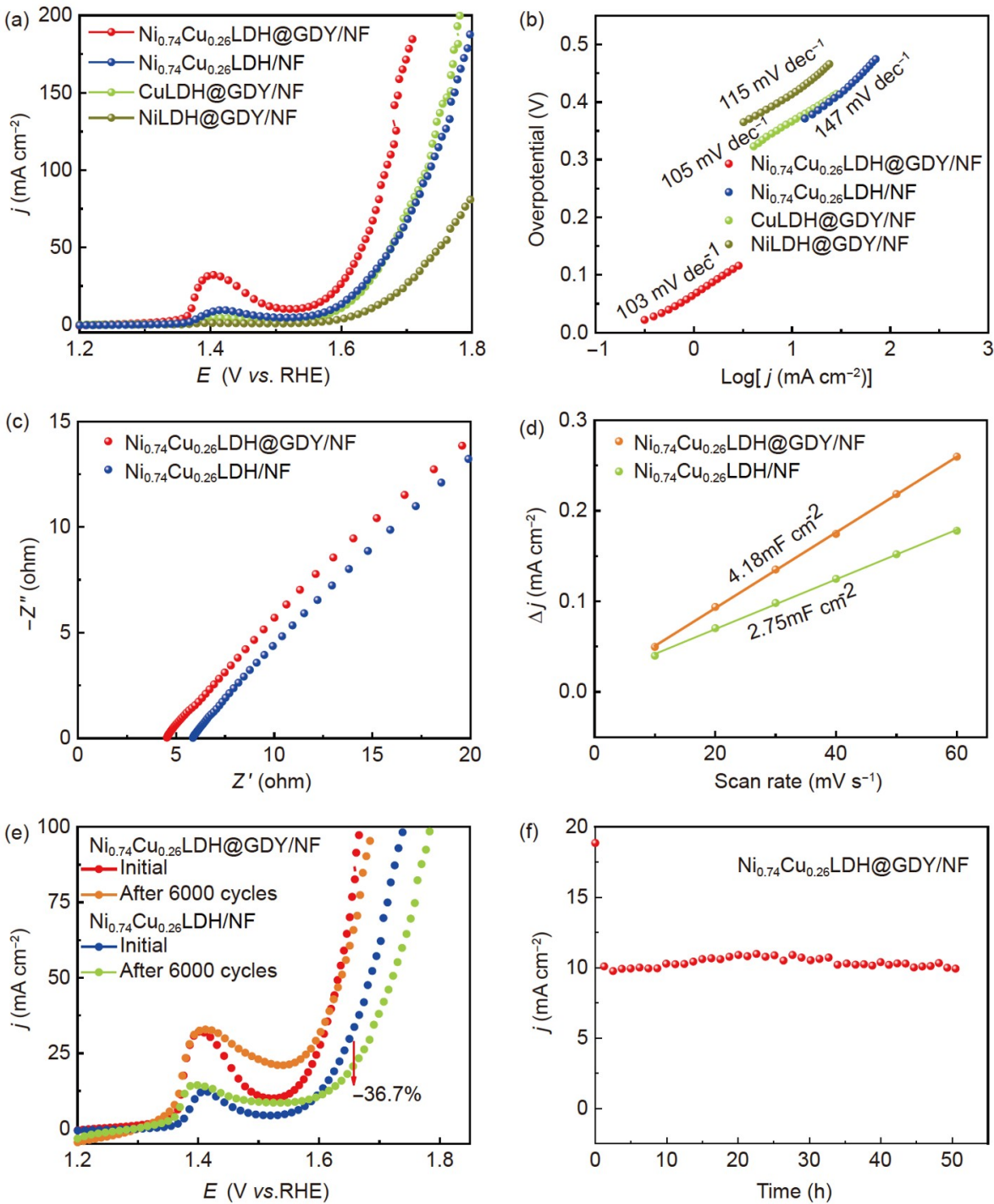

图 5 (a) 极化曲线; (b) Tafel斜率; (c) Nyquist曲线; (d) 双电层电容 $\left(C_{\mathrm{d})}\right.$ )值; (e) 6000圈循环测试前后的极化曲线; (f) $i-t$ 曲线 (网 络版彩图)

Figure 5 (a) Polarization curves and (b) corresponding Tafel slopes of the as-prepared samples in $1.0 \mathrm{M} \mathrm{KOH}$; (c) Nyquist plots of EIS for the samples; (d) dependence of the capacitive current on the scan rates; (e) polarization curves of $\mathrm{Ni}_{0.74} \mathrm{Cu}_{0.26} \mathrm{LDH} @ \mathrm{GDY} / \mathrm{NF}, \mathrm{Ni} \mathrm{i}_{0.74} \mathrm{Cu} 0.26 \mathrm{LDH} / \mathrm{NF}$ before and after stability tests in $1.0 \mathrm{M} \mathrm{KOH}$; (f) current density-time curves for $\mathrm{Ni}_{0.74} \mathrm{Cu}_{0.26} \mathrm{LDH} @$ GDY/NF (color online).

直径对应电荷转移电阻 $\left(R_{\mathrm{ct}}\right)$, 与 $\mathrm{Ni}_{0.74} \mathrm{Cu}_{0.26} \mathrm{LDH} / \mathrm{NF}$ 相 比, $\mathrm{Ni}_{0.74} \mathrm{Cu}_{0.26} \mathrm{LDH} @ \mathrm{GDY} / \mathrm{NF}$ 的阻抗谱中半圆直径更 小, 具有更小的电荷转移电阻, 这可能是因为石墨炔基 异质结构的形成加速电荷转移引起的. 电化学活性面 积 $(\mathrm{ECSA})$ 的大小与电化学双电层电容 $\left(C_{\mathrm{d} 1}\right.$ ) 数值成比 例关系. 因此, 可以通过实验测定并计算 $C_{\mathrm{dl}}$, 以此来评 价电化学活性面积的大小. 具体实验参数测试如图 $5 \mathrm{~d}$ 所示，在 $0.1 \mathrm{~V}$ 电压区间范围内进行不同扫描速度的循 环伏安法(CV)测试, 经计算, $\mathrm{Ni}_{0.74} \mathrm{Cu}_{0.26} \mathrm{LDH} @ \mathrm{GDY} /$
$\mathrm{NF}$ 和 $\mathrm{Ni}_{0.74} \mathrm{Cu}_{0.26} \mathrm{LDH} / \mathrm{NF}$ 的双电层电容值分别为 4.18 和2.75 mF cm ${ }^{-2} \cdot \mathrm{Ni}_{0.74} \mathrm{Cu}_{0.26} \mathrm{LDH} @ \mathrm{GDY} / \mathrm{NF}$ 的双电层 电容值大于 $\mathrm{Ni}_{0.74} \mathrm{Cu}_{0.26} \mathrm{LDH} / \mathrm{NF}$, 这说明石墨炔的引入 可以增加活性位点的数目, 提高催化性能.

催化剂稳定性是表征催化剂好坏的关键性参数, 尤其在实际工业生产中关系着催化剂的有效性和持续 性. 我们通过CV法和 $i-t$ 法分别对催化剂的稳定性进行 了表征. 实验结果(图5e)显示, $\mathrm{Ni}_{0.74} \mathrm{Cu}_{0.26} \mathrm{LDH} @$ GDY/ $\mathrm{NF}$ 的催化活性在经过 6000 圈连续循环测试后几乎无 
衰减. 而纯 $\mathrm{Ni}_{0.74} \mathrm{Cu}_{0.26} \mathrm{LDH} / \mathrm{NF}$ 的催化活性则衰减了将 近 $36.7 \%$. 此外, 如图 $5 \mathrm{f}$ 所示, 在经过 $51 \mathrm{~h}$ 恒电流测试 后, $\mathrm{Ni}_{0.74} \mathrm{Cu}_{0.26} \mathrm{LDH} @$ GDY/NF电催化性能几乎无衰减. 通过SEM分析(补充图9), 经过长期稳定性测试后, $\mathrm{Ni}_{0.74} \mathrm{Cu}_{0.26} \mathrm{LDH} @$ GDY/NF的形貌得到保持, 说明了 $\mathrm{Ni}_{0.74} \mathrm{Cu}_{0.26} \mathrm{LDH} @$ GDY/NF良好的稳定性.

\section{4 结论}

本文中，我们通过石墨炔在铜双金属氢氧化物表 面原位生长的策略成功制备石墨炔基异质结构催化 剂. 研究结果显示, 通过改变 $\mathrm{Cu} / \mathrm{Ni}$ 的元素比例, 可以有
效调控催化剂的结构形貌以及OER催化活性. $\mathrm{Ni}_{0.74^{-}}$ $\mathrm{Cu}_{0.26} \mathrm{LDH} @ \mathrm{GDY} / \mathrm{NF}$ 具有最佳的碱性OER催化活性. 我们的研究结果表明, 石墨炔在催化剂异质结构设计 中发挥至关重要的作用: (1) 石墨炔异质结理想界面 结构的形成有利于产生新的活性位点, 增加活性位点 数量; (2) 石墨炔与活性位点之间的高效电荷转移有 利于提高催化剂的导电性; (3)石墨炔天然大孔结构将 有利于物质输运以及气体产物的释放, 促进反应进行; (4) 石墨炔的高的化学稳定性可以有效保护催化剂不 被腐蚀, 提高其催化稳定性. 我们的研究显示了石墨 炔在可控制备理想界面结构中的天然优势，为开发新 型催化剂和新能源转换器件提供了新思路.

\section{补充材料}

本文的补充材料见网络版chemen.scichina.com. 补充材料为作者提供的原始数据, 作者对其学术质量和内容负责.

\section{参考文献}

1 Hestand NJ, Kazantsev RV, Weingarten AS, Palmer LC, Stupp SI, Spano FC. J Am Chem Soc, 2016, 138: 11762-11774

2 Du J, Li F, Sun L. Chem Soc Rev, 2021, 50: 2663-2695

3 Tian L, Zhai X, Wang X, Li J, Li Z. J Mater Chem A, 2020, 8: 14400-14414

4 Zhou Y, Che F, Liu M, Zou C, Liang Z, De Luna P, Yuan H, Li J, Wang Z, Xie H, Li H, Chen P, Bladt E, Quintero-Bermudez R, Sham TK, Bals S, Hofkens J, Sinton D, Chen G, Sargent EH. Nat Chem, 2018, 10: 974-980

5 Zhang B, Zhang J, Hua M, Wan Q, Su Z, Tan X, Liu L, Zhang F, Chen G, Tan D, Cheng X, Han B, Zheng L, Mo G. J Am Chem Soc, 2020, 142: $13606-13613$

6 Cui Z, Li Y, Fu G, Li X, Goodenough JB. Adv Mater, 2017, 29: 1702385

7 Cheng F, Chen J. Chem Soc Rev, 2012, 41: 2172-2192

8 Wang Y, Wang S, Zhang SL, Lou XWD. Angew Chem Int Ed, 2020, 59: 11918-11922

9 Zhao JW, Li CF, Shi ZX, Guan JL, Li GR. Research, 2020, 2020: 1-15

10 Xu H, Shi ZX, Tong YX, Li GR. Adv Mater, 2018, 30: 1705442

11 Zhou Z, Pei Z, Wei L, Zhao S, Jian X, Chen Y. Energy Environ Sci, 2020, 13: 3185-3206

12 Zheng Y, Jiao Y, Vasileff A, Qiao SZ. Angew Chem Int Ed, 2018, 57: 7568-7579

13 Sun H, Yan Z, Liu F, Xu W, Cheng F, Chen J. Adv Mater, 2020, 32: 1806326

14 Hui L, Xue Y, Huang B, Yu H, Zhang C, Zhang D, Jia D, Zhao Y, Li Y, Liu H, Li Y. Nat Commun, 2018, 9: 5309

15 Zhao S, Xue Y, Wang Z, Zheng Z, Luan X, Gao Y, Li Y. Mater Chem Front, 2021, 5: 4153-4159 
Gao X, Li J, Du R, Zhou J, Huang MY, Liu R, Li J, Xie Z, Wu LZ, Liu Z, Zhang J. Adv Mater, 2017, 29: 1605308

Matsuoka R, Sakamoto R, Hoshiko K, Sasaki S, Masunaga H, Nagashio K, Nishihara H. J Am Chem Soc, 2017, 139: 3145-3152

Li J, Gao X, Li Z, Wang JH, Zhu L, Yin C, Wang Y, Li XB, Liu Z, Zhang J, Tung CH, Wu LZ. Adv Funct Mater, 2019, 29: 1808079 Han YY, Lu XL, Tang SF, Yin XP, Wei ZW, Lu TB. Adv Energy Mater, 2018, 8: 1702992

Gao X, Zhu Y, Yi D, Zhou J, Zhang S, Yin C, Ding F, Zhang S, Yi X, Wang J, Tong L, Han Y, Liu Z, Zhang J. Sci Adv, 2018, 4: eaat6378

Yan H, Yu P, Han G, Zhang Q, Gu L, Yi Y, Liu H, Li Y, Mao L. Angew Chem Int Ed, 2019, 58: 746-750

Xue Y, Guo Y, Yi Y, Li Y, Liu H, Li D, Yang W, Li Y. Nano Energy, 2016, 30: 858-866

Xue Y, Zuo Z, Li Y, Liu H, Li Y. Small, 2017, 13: 1700936

Xue Y, Huang B, Yi Y, Guo Y, Zuo Z, Li Y, Jia Z, Liu H, Li Y. Nat Commun, 2018, 9: 1460

Yu H, Xue Y, Hui L, Zhang C, Li Y, Zuo Z, Zhao Y, Li Z, Li Y. Adv Mater, 2018, 30: 1707082

Yu H, Xue Y, Hui L, Zhang C, Zhao Y, Li Z, Li Y. Adv Funct Mater, 2018, 28: 1707564

Hui L, Xue Y, He F, Jia D, Li Y. Nano Energy, 2019, 55: 135-142

Hui L, Xue Y, Yu H, Liu Y, Fang Y, Xing C, Huang B, Li Y. J Am Chem Soc, 2019, 141: 10677-10683

Xue Y, Hui L, Yu H, Liu Y, Fang Y, Huang B, Zhao Y, Li Z, Li Y. Nat Commun, 2019, 10: 2281

Yu H, Xue Y, Huang B, Hui L, Zhang C, Fang Y, Liu Y, Zhao Y, Li Y, Liu H, Li Y. iScience, 2019, 11: 31-41

Yu H, Xue Y, Hui L, He F, Zhang C, Liu Y, Fang Y, Xing C, Li Y, Liu H, Li Y. Nano Energy, 2019, 64: 103928

Fang Y, Xue Y, Li Y, Yu H, Hui L, Liu Y, Xing C, Zhang C, Zhang D, Wang Z, Chen X, Gao Y, Huang B, Li Y. Angew Chem Int Ed, 2020, 59: 13021-13027

Fang Y, Xue Y, Hui L, Yu H, Li Y. Angew Chem Int Ed, 2021, 60: 3170-3174

Du Y, Xue Y, Zhang C, Liu Y, Fang Y, Xing C, He F, Li Y. Adv Energy Mater, 2021, 11: 2100234

Wang Z, Zheng Z, Xue Y, He F, Li Y. Adv Energy Mater, 2021, 11: 2101138

Du Y, Zhou W, Gao J, Pan X, Li Y. Acc Chem Res, 2020, 53: 459-469

Xue Y, Li Y, Zhang J, Liu Z, Zhao Y. Sci China Chem, 2018, 61: 765-786

Yu H, Xue Y, Li Y. Adv Mater, 2019, 31: 1803101

Zuo Z, Wang D, Zhang J, Lu F, Li Y. Adv Mater, 2019, 31: 1803762

Tong Y, Yu X, Shi G. Phys Chem Chem Phys, 2017, 19: 4821-4826 


\title{
Two-dimensional graphdiyne/metal hydroxide heterojunction for high-efficiency oxygen evolution reaction
}

\author{
Yaqi $\mathrm{Gao}^{1}$, Yurui Xue ${ }^{1 *}$, Yuliang $\mathrm{Li}^{1,2^{*}}$ \\ ${ }^{1}$ Science Center for Material Creation and Energy Conversion, School of Chemistry and Chemical Engineering, Shandong University, Jinan 250100, \\ China \\ ${ }^{2}$ Institute of Chemistry, Chinese Academy of Sciences, Beijing 100190, China \\ *Corresponding authors (email: xueyurui@iccas.ac.cn; ylli@iccas.ac.cn)
}

\begin{abstract}
Oxygen evolution reaction (OER) is an important reaction in energy conversion process. It is of great significance to develop efficient, stable and low-cost catalysts for OER. Herein, we report a simple method for the insitu growth of graphdiyne (GDY) films on the surface of metal hydroxides, forming a new type of GDY/metal hydroxide heterojunction interface structure. By changing the chemical composition of the metal species, the improved OER activity and stability were achieved. Our results show that the $\mathrm{Ni}_{0.74} \mathrm{Cu}_{0.26} \mathrm{LDH} @$ GDY/NF has the best OER catalytic performance with the low overpotential of $292 \mathrm{mV}$ at the current density of $10 \mathrm{~mA} \mathrm{~cm}^{-2}$, and excellent long-term stability. Experimental results reveal that the introduction of GDY can effectively increase the number of the active sites, improve the conductivity of the catalyst, protect the catalyst from corrosion, and finally enhance the overall catalytic performance.
\end{abstract}

Keywords: graphdiyne, heterojunction catalyst, metal hydroxide, oxygen evolution reaction

doi: $10.1360 /$ SSC-2021-0203 